\title{
REMARKS ON THE STRING OF BEADS
}

\author{
DONALD R. WILKEN
}

1. Introduction. Let $D$ be the closed unit disc in the complex plane $C, D=\{z:|z| \leqq 1\}$. Let $K$ be a closed subset of the interval $[-1,1]$. If $I$ is any interval component of $[-1,1]-K$, remove from $D$ the open disc with $I$ as a diameter. Let $X$ be the compact set which remains when all such open discs have been deleted. In the case where $K$ has no interior relative to $[-1,1], X$ is referred to as the "string of beads" example. Let $R(X)$ denote the function algebra consisting of the functions which are uniform limits on $X$ of rational functions with poles off $X$. The string of beads example has been studied with regard to the question of the connectivity of the Gleason parts of $R(X)$ for an arbitrary compact plane set $X$. We refer the reader to [1], [4] and [5] for more detailed discussions of all of the concepts discussed below.

It can be shown (an outline of the proof is in $\$ 2$ ) that, when $K$ has zero linear measure, the upper and lower components of the interior of $X$, denoted $U$ and $L$, respectively, lie in distinct parts of $R(X)$. Moreover, each point of $K$ is a peak point for $R(X)$. When $K$ has positive linear measure it is easy to see that both $U$ and $L$ lie in the same part. In [3] Hoffman conjectured that in this case there is always some point of $K$ which is in the same part as $U$ and $L$ so that the part is connected. Since it is known that when a point of $K$ fails to be a peak point then it belongs to the nontrivial part of $R(X)$, it suffices to show some point of $K$ is not a peak point. The purpose of this note is to show that in many cases Hoffman's conjecture is correct and that, moreover, in these cases, almost every point of $K$ belongs to the nontrivial part.

The remarkable new fact, however, is that A. M. Davie of the University of Dundee, Dundee, Scotland has constructed a string of beads example for which $K$ has positive linear measure but each point of $K$ is a peak point. Hence $U$ and $L$ separate the part containing them and exhibit the first example of a disconnected part for $R(X)$. John Garnett of UCLA has constructed a similar example independently. With these startling examples in mind, we record our theorem with its proof merely as an interesting observation about the string of beads examples.

Presented to the Society, January 24, 1969 under the title Some remarks on the string of beads; received by the editors February 6, 1969. 
2. Some details. As promised in the introduction we begin with a sketch of the proof of the fact that, when $K$ has zero linear measure, $U$ and $L$ lie in different parts. It follows from a theorem of Melnikov (see [5, p. 112, Theorem 14.1]) that for any string of beads example, $R(X)=A(X)$, where $A(X)$ denotes the algebra of all continuous functions of $X$ which are analytic on the interior of $X$. But it is an easy consequence of a theorem of Fatou and its proof (see [2, p. 80]) that there is a function in $A(X)$ with $f \equiv 1$ on $U$ and $|f|<1$ on $L$. The existence of such a function in $R(X)$ is sufficient to distinguish the parts containing $U$ and $L$. When $K$ has positive linear measure, harmonic measure for a point in $U$ is not singular with respect to harmonic measure for a point in $L$-hence $L$ and $U$ lie in the same part (see Chapter VI of [1]).

Our observation about the string of beads is the following. For the $n$th deleted disc, let $r_{n}$ denote its radius, let $C_{n}$ denote its boundary, and let $x_{n}$ denote its center.

TheOREM. If $\sum_{n=1}^{\infty} r_{n}<1$ and $\sum_{n=1}^{\infty} \sqrt{ } r_{n}<\infty$, then almost every point of $K$ with respect to linear measure is not a peak point for $R(X)$. (The first condition is, of course, equivalent to $K$ having positive linear measure.)

COROLLARY. Under the hypotheses of the theorem, the part containing $U$ and $L$ is connected and contains almost every point of $K$.

Proof of Corollary. A point of $X$ is not a peak point if and only if the part containing it has positive planar Lebesgue measure (see $[4])$.

To prove the theorem we use the following well-known lemma.

Lemma. Let $t \in K$. Let $a_{n}=\left|t-x_{n}\right|$. If $\sum_{n=1}^{\infty} r_{n} / a_{n}<\infty$, then $t$ is not a peak point of $R(X)$.

Proof of Lemma. To show $t$ is not a peak point it suffices to exhibit a nontrivial finite (complex) representing measure for $t$. Define a measure $\mu$ on the boundary of $X$ by

$$
\mu=\frac{1}{2 \pi i} \frac{d z}{z-t} \quad \text { on }|z|=1
$$

and

$$
\mu=\frac{1}{2 \pi i} \frac{-d z}{z-t} \quad \text { on } C_{n}, n=1,2, \cdots
$$


It is an elementary computation to show the condition

$$
\sum_{n=1}^{\infty} \frac{r_{n}}{a_{n}}<\infty
$$

guarantees that $\mu$ is a finite measure. The fact that it represents is an immediate consequence of the Cauchy integral formula.

Proof of Theorem. Let $\epsilon>0$ satisfy $\sum_{n=1}^{\infty} r_{n}+\epsilon<1$. Since $\sum_{n=1}^{\infty} \sqrt{ } r_{n}<\infty$ we can choose positive numbers $b_{n}$ such that $b_{n} \leqq 1$ for all $n$,

$$
\sum_{n=1}^{\infty} \frac{r_{n}}{b_{n}}<\sum_{n=1}^{\infty} r_{n}+\epsilon \text { and } \sum_{n=1}^{\infty} b_{n}<\infty .
$$

Let $K_{\epsilon}$ be the set left in $[-1,1]$ after removing from $D$ discs of radius $r_{n} / b_{n}$ with centers $x_{n}$. Then $K_{\epsilon} \subset K$ and almost every point of $K$ lies in some $K_{\epsilon}$, where $0<\epsilon<1-\sum_{n=1}^{\infty} r_{n}$. Now if $t \in K_{\epsilon}$, some $\epsilon$, then $\left|t-x_{n}\right| \geqq r_{n} / b_{n}$. Hence, if $a_{n}=\left|t-x_{n}\right|, \quad \sum_{n=1}^{\infty}\left(r_{n} / a_{n}\right) \leqq \sum_{n=1}^{\infty} b_{n}$ $<\infty$. By the lemma $t$ is not a peak point of $R(X)$.

REMARK. Since the end points of the intervals complementary to $K$ are peak points, "almost every" cannot be replaced by "every" in the statement of the theorem.

\section{REFERENCES}

1. T. W. Gamelin, Uniform algebras, Prentice-Hall, Englewood Cliffs, N. J., 1969.

2. K. Hoffman, Banach spaces of analytic functions, Prentice-Hall, Englewood Cliffs, N. J., 1962.

3. - "Parts and analyticity," in Function algebras, edited by F. T. Birtel, Scott, Foresman and Co., Glenview, Ill., 1966, pp. 1-5.

4. D. Wilken, Lebesgue measure of parts for $R(X)$, Proc. Amer. Math. Soc. 18 (1967), 508-512.

5. L. Zalcman, Analytic capacity and rational approximation, Lecture Notes in Mathematics, No. 50, Springer-Verlag, Berlin, 1968.

State University of New York at Albany 